\title{
$355 \mathrm{~nm}$ 激光烧蚀 $\operatorname{LiMn}_{2} \mathrm{O}_{4}$ 反应产物的 飞行时间质谱
}

\author{
赵胜利 刘先年 秦启宗*
}

(复旦大学化学系激光化学研究所, 上海 200433)

\begin{abstract}
摘要采用角分辨飞行时间质谱法研究了 $355 \mathrm{~nm}$ 脉冲激光烧蚀 $\mathrm{LiMn}_{2} \mathrm{O}_{4}$ 的反应. 在 较低激光能量密度 $\left(0.8 \mathrm{~J} \cdot \mathrm{cm}^{-2}\right)$ 测得的离子和中性烧蚀产物主要有 $\mathrm{Li}, \mathrm{O}, \mathrm{LiO}, \mathrm{LiO}_{2}, \mathrm{Mn}$, $\mathrm{Li}_{2}, \mathrm{Li}_{4}, \mathrm{Li}_{6}, \mathrm{LiMn}, \mathrm{MnO}, \mathrm{MnO}_{2}$ 等. 激光能量密度较大时, 烧蚀产物中的氧化物不仅相 对量增加，而且物种更加丰富。它们的飞行时间谱可用带质心速度的 Maxwell-Boltzmann 分布函数拟合. 烧蚀产物 $\mathrm{Li}, \mathrm{LiO}, \mathrm{LiO}_{2}$ 和 $\mathrm{Mn}$ 存在能量密度表观阈 值, 并且离子产物的阈值比相应的中性产物高. 烧蚀产物中原生离子和中性产物的空 间角分布可用 $\cos ^{n} \theta$ 或 $a \cos \theta+(1-a) \cos ^{n} \theta$ 拟合. 此外，对 $355 \mathrm{~nm}$ 脉冲激光对 $\mathrm{LiMn}_{2} \mathrm{O}_{4}$ 的烧蚀机理进行了讨论.
\end{abstract}

\section{关键词 $\mathrm{LiMn}_{2} \mathrm{O}_{4}$ 激光烧蚀 TOF 角分布}

全固态薄膜锂离子电池具有能量密度高, 循环性能和安全性能好等优点, 近年来对此类 电池的研究非常活跃 ${ }^{[1]}$. 其中正极活性材料的研究是全固态薄膜锂离子电池研究的一个重要 部分. $\mathrm{LiMn}_{2} \mathrm{O}_{4}$ 和 $\mathrm{LiNiO}_{2}, \mathrm{LiCoO}_{2}$ 相比具有理论容量高, 价格便宜, 毒性小等优点, 被认为是一 种很有应用前景的正极材料 ${ }^{[2]}$. 文献中报道了不同的制备 $\mathrm{LiMn}_{2} \mathrm{O}_{4}$ 薄膜的方法, 如射频磁控溅 射沉积法、电子束沉积法、脉冲激光沉积法(pulsed laser deposition, PLD)等 ${ }^{[3]}$. 由于 PLD 法具 有沉积率高, 并且较容易获得具有与靶保持相同化学计量比的高质量 $\mathrm{LiMn}_{2} \mathrm{O}_{4}$ 薄膜, 因此受 到人们的重视. 目前采用 PLD 对 $\mathrm{LiMn}_{2} \mathrm{O}_{4}$ 薄膜的研究已有不少报道 ${ }^{[4,5]}$, 但这些研究主要局限 于薄膜的制备过程和电化学性质等方面, 而对激光烧蚀 $\mathrm{LiMn}_{2} \mathrm{O}_{4}$ 靶产生的反应产物及其能量 和空间分布很少报道.

我们曾采用 PLD 法成功地制备了一些锂离子电池薄膜电极以及具有电致变色、高介电常 数和光致发光性质的薄膜材料. 为了进一步理解激光烧蚀机理, 我们还系统地研究了中性和 离子烧蚀产物, 并且考察了它们的动能和空间角分布 ${ }^{[6 ~ 10]}$. 研究激光烧蚀产物动能和空间角分 布可以给烧蚀羽状物动力学以及薄膜的化学组成和均匀性提供有用的信息. 本文将采用角分 辨和时间分辨的质谱技术, 对 $355 \mathrm{~nm}$ 激光烧蚀 $\mathrm{LiMn}_{2} \mathrm{O}_{4}$ 靶的反应产物的质量分布, 飞行时间 谱(time of flight, TOF)以及产物的空间角分布进行研究, 并探讨了脉冲激光对 $\mathrm{LiMn}_{2} \mathrm{O}_{4}$ 的烧蚀 机理. 


\section{1 实验部分}

用于角分辨和时间分辨质谱测量的实验装置详见前文 ${ }^{[11]} .355 \mathrm{~nm}$ 激光由 Nd/YAG 激光器

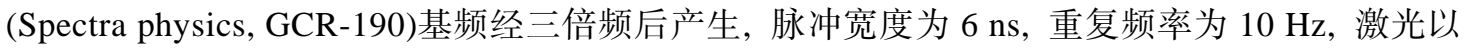
$45^{\circ}$ 的人射角辐照靶表面, 激光功率由热电功率计测定. 在真空反应室中心安装一个可绕轴转 动的样品架, 样品架上的靶子由 $\mathrm{LiMn}_{2} \mathrm{O}_{4}$ 粉末压片, 在 $1073 \mathrm{~K}$ 下焙烧 $5 \mathrm{~h}$ 后制成. 经脉冲激光 烧蚀靶子后反应产物的质量和空间角分布用一可绕反应中心转动的四极质谱仪(QMS, ULVAC MSQ-400)测定, 信号经前置放大后输人 $20 \mathrm{MHz}$ 的瞬态记录仪, 瞬态记录的触发信号由脉冲 激光提供. 产物的相对产额由其飞行时间谱的积分面积给出, 测定产物角分布的探测角范围 为 $0 \sim 90^{\circ}$. 产物从样品表面至 QMS 电离区中心的飞行距离为 $18 \mathrm{~cm}$. 在测定产物中的原生离 子时, 将 QMS 电离器内用以发射电子的电离灯丝关闭; 测量中性产物时将电离灯丝开启, 由 $70 \mathrm{eV}$ 电子轰击使中性产物电离, 这时测得的离子信号来源于原生离子和中性产物, 扣除原生 离子的贡献, 即可得中性产物得信号. 由于产物粒子在四极杆内有一定的漂流时间, 在数据处 理时必须给予校正, 在本实验条件下漂流时间 $\Delta t \sim 4.15 \mathrm{~m}^{1 / 2} \mu \mathrm{s}^{[12]}$, 其中 $\mathrm{m}$ 是粒子的质量.

\section{2 结果与讨论}

\section{1 烧蚀产物的质量分布和 TOF 谱}

图 1 是 $355 \mathrm{~nm}$ 脉冲激光烧蚀 $\mathrm{LiMn}_{2} \mathrm{O}_{4}$ 生成的原生离子产物和中性产物的质量分布, 其中
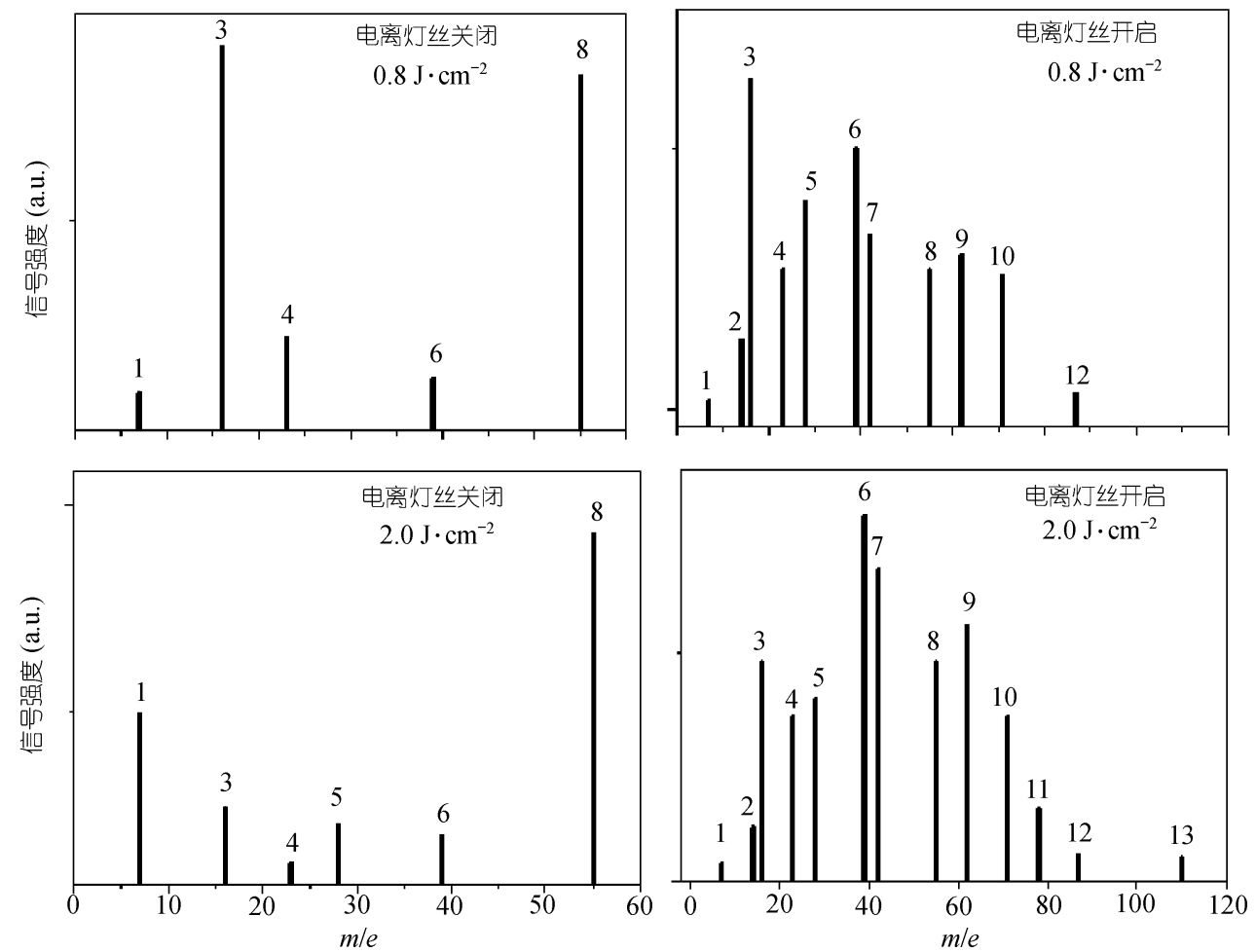

图 1 在激光能量密度为 0.8 和 $2.0 \mathrm{~J} \cdot \mathrm{cm}^{-2}$, 电离灯丝关闭和开启时测得 $355 \mathrm{~nm}$ 脉冲 激光烧蚀 $\mathrm{LiMn}_{2} \mathrm{O}_{4}$ 产物的质量分布 1 13 分别为: $\mathrm{Li}, \mathrm{Li}_{2}, \mathrm{O}, \mathrm{LiO}, \mathrm{Li}_{4}, \mathrm{LiO}_{2}, \mathrm{Li}_{6}, \mathrm{Mn}, \mathrm{LiMn}, \mathrm{MnO}, \mathrm{LiMnO}, \mathrm{MnO}_{2}, \mathrm{Mn}_{2}$ 
$\mathrm{O}_{2}$ 的相对强度太强而未被列出. 可以看出, 在激光能量密度为 $0.8 \mathrm{~J} \cdot \mathrm{cm}^{-2}$ 时, 测得的烧蚀产物 有: 离子和中性的 $\mathrm{Li}(m / e=7), \mathrm{O}(m / e=16), \mathrm{LiO}(m / e=23), \mathrm{LiO}_{2}(m / e=39), \mathrm{Mn}(m / e=55)$ 以 及中性的 $\mathrm{Li}_{4}(m / e=28), \mathrm{Li}_{6}(m / e=42), \mathrm{LiMn}(m / e=62), \mathrm{MnO}(m / e=71), \mathrm{MnO}_{2}(m / e=87)$ 等. 然 而, 在更高的激光能量密度 $\left(2.0 \mathrm{~J} \cdot \mathrm{cm}^{-2}\right)$ 时, 除了上述测得的产物之外, 还观测到其他不常见的 离子和中性产物, 如 $\mathrm{LiMnO}(m / e=78), \mathrm{Mn}_{2}(m / e=110)$ 等.

从上述的烧蚀产物的质量分布可以看出, 中性产物比离子产物丰富, 这是激光烧蚀氧化 物的一般特征. 由于溅射出来的粒子中氧含量很高, 并呈气态逃逸, 会导致薄膜缺氧. 因此, PLD 制备 $\mathrm{LiMn}_{2} \mathrm{O}_{4}$ 薄膜时通常在环境气氛中补充一定量的氧. 另外, 在激光能量密度较大时, 烧蚀产物中的氧化物不仅相对量增加, 而且物种更加丰富. 这意味着较高的激光能量密度对 于团簇和其他化合物分子的形成是有利的.
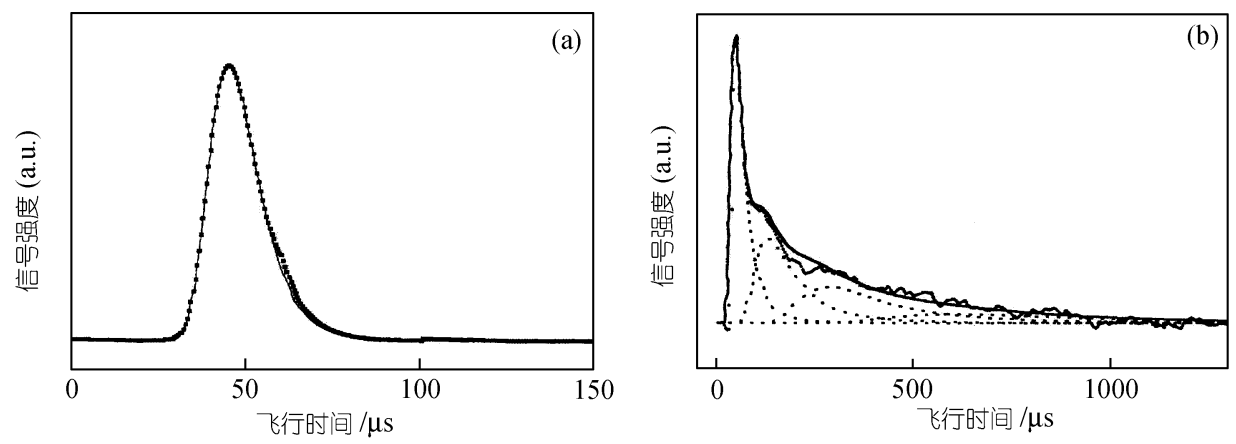

图 $2 \mathrm{Mn}$ 的 TOF 谱以及 M-B 拟合结果

(a) 电离灯丝关闭; (b) 电离灯丝开启. 点为实验测量结果, 虚线为 M-B 拟合获得的不同组分, 实线为拟合结果

$\mathrm{LiMn}_{2} \mathrm{O}_{4}$ 靶在脉冲激光的作用下, 大量的烧蚀产物将同时离开靶表面向真空绝热膨胀, 并 在表面附近经多次碰撞后获得一个定向的速度, 通常它们的 TOF 谱可用带质心速度的 Maxwell-Boltzmann(M-B)分布函数来拟合 ${ }^{[12]}$ :

$$
F(t)=A t^{-4} \exp \left(-b_{1} t^{-2}+b_{2} t^{-1}\right),
$$

其中 $A, b_{1}$ 以及 $b_{2}$ 是拟合参数 $\left(b_{1}=m L^{2} / 2 k T_{S}, b_{2}=2 b_{1} u / l\right), t$ 是飞行时间, $u$ 是质心速度, $l$ 是飞行 距离, $k$ 是 Boltzmann 常数, $T_{S}$ 为产物离开靶表面时的温度. 如果信号来源多于一个母体时, 可 用多组分 M-B 分布来拟合:

$$
F(t)=\sum_{i=1}^{p} F_{i}(t)=\sum_{i=1}^{p} A_{i} t^{-4} \exp \left(-b_{1 i} t^{-2}+b_{2 i} t^{-1}\right),
$$

其中 $p$ 为母体的数目.

我们对激光烧蚀 $\mathrm{LiMn}_{2} \mathrm{O}_{4}$ 的主要产物的 TOF 谱分别进行了测定, 这里仅给出一个具有代 表性的 TOF 谱, 即 $355 \mathrm{~nm}$ 激光在能量密度 $2.0 \mathrm{~J} \cdot \mathrm{cm}^{-2}$ 烧蚀 $\mathrm{LiMn}_{2} \mathrm{O}_{4}$ 时所得锰的 TOF 谱. 由 图 2 可见, 在电离灯丝关闭时仅有一个峰; 而在电离灯丝开启时 TOF 谱比较复杂, 由一个快 峰和具有很宽的拖尾峰组成. 通过对上述两种情况下测得的 $\mathrm{Mn}^{+}$的 TOF 谱进行 M-B 拟合. 当 电离灯丝关闭时, $\mathrm{Mn}^{+}$能很好地用单组分 $\mathrm{M}-\mathrm{B}$ 分布拟合, 其峰值时间为 $37.1 \mu \mathrm{s}$, 表明此时 $\mathrm{Mn}^{+}$离子信号来源单一, 是原生锰离子的贡献; 当电离灯丝开启时, 由于 $\mathrm{Mn}^{+}$的 TOF 谱有很 宽的拖尾, 须用多组分 M-B 分布拟合. 拟合结果表明, 电离灯丝开启时有 4 个峰组成(包括 1 
个快峰和 3 个慢峰), 其中快峰可认为是来源于烧蚀产物中的原生离子, 这可从灯丝关时测得 的 TOF 谱的峰位时间 $\left(t_{m}=37.1 \mu \mathrm{s}\right)$ 及拟合曲线得到印证; 而拖尾中的 3 个慢峰的峰值时间分 别为 123,289 和 $607 \mu \mathrm{s} .123 \mu \mathrm{s}$ 峰可归于中性 $\mathrm{Mn}$ 原子的贡献, 而后两个峰则可能是 $\mathrm{MnO}$ 和 $\mathrm{LiMnO}$ 在电离室内经电子轰击裂解生成的 $\mathrm{Mn}^{+}$的贡献. 从 TOF 谱的拟合结果可算出原生锰 离子和锰原子的最可几平动能分别为 6.71 和 $0.61 \mathrm{eV}$, 离开靶表面的温度为 $3.9 \times 10^{4}$ 和 $6.1 \times$ $10^{3} \mathrm{~K}$, 而它们的质心速度分别为 $2.7 \times 10^{3}$ 和 $2.2 \times 10^{2} \mathrm{~m} / \mathrm{s}$.

表 1 在激光能量密度 $2.0 \mathrm{~J} \cdot \mathrm{cm}^{-2}$ 时, $355 \mathrm{~nm}$ 脉冲激光烧蚀 $\mathrm{LiMn}_{2} \mathrm{O}_{4}$ 产物的 TOF 谱的拟合结果

\begin{tabular}{|c|c|c|c|c|c|c|c|c|}
\hline \multirow{2}{*}{ 产物 } & \multicolumn{4}{|c|}{ 原生离子 } & \multicolumn{4}{|c|}{ 中性产物 } \\
\hline & $t_{m} / \mu \mathrm{s}$ & $K_{E} / \mathrm{eV}$ & $u / \mathrm{m} \cdot \mathrm{s}^{-1}$ & $T_{S} / \mathrm{K}$ & $t_{m} / \mu \mathrm{s}$ & $K_{E} / \mathrm{eV}$ & $u / \mathrm{m} \cdot \mathrm{s}^{-1}$ & $T_{S} / \mathrm{K}$ \\
\hline $\mathrm{Li}$ & 21.3 & 2.59 & $2.3 \times 10^{3}$ & $2.6 \times 10^{4}$ & 102 & 0.11 & $2.7 \times 10^{2}$ & $4.9 \times 10^{3}$ \\
\hline $\mathrm{O}$ & 35.2 & 2.17 & $1.4 \times 10^{3}$ & $3.1 \times 10^{4}$ & 124 & 0.17 & $3.8 \times 10^{2}$ & $7.4 \times 10^{3}$ \\
\hline $\mathrm{LiO}$ & 23.9 & 6.74 & $1.7 \times 10^{3}$ & $4.0 \times 10^{4}$ & 157 & 0.16 & $2.6 \times 10^{2}$ & $8.9 \times 10^{3}$ \\
\hline $\mathrm{LiO}_{2}$ & 39.2 & 4.26 & $2.1 \times 10^{3}$ & $6.1 \times 10^{4}$ & 171 & 0.22 & $4.1 \times 10^{2}$ & $6.3 \times 10^{3}$ \\
\hline $\mathrm{Mn}$ & 37.1 & 6.71 & $2.7 \times 10^{3}$ & $3.9 \times 10^{4}$ & 123 & 0.61 & $2.2 \times 10^{2}$ & $6.1 \times 10^{3}$ \\
\hline $\mathrm{Li}_{4}$ & 31.5 & 4.74 & $2.9 \times 10^{3}$ & $1.3 \times 10^{4}$ & 130 & 0.28 & $3.1 \times 10^{2}$ & $5.4 \times 10^{3}$ \\
\hline $\mathrm{Li}_{2}$ & & & & & 122 & 0.16 & $2.6 \times 10^{2}$ & $3.1 \times 10^{3}$ \\
\hline $\mathrm{Li}_{6}$ & & & & & 161 & 0.18 & $2.7 \times 10^{2}$ & $4.2 \times 10^{3}$ \\
\hline LiMn & & & & & 192 & 0.28 & $3.1 \times 10^{2}$ & $2.7 \times 10^{3}$ \\
\hline $\mathrm{MnO}$ & & & & & 173 & 0.40 & $3.5 \times 10^{2}$ & $4.9 \times 10^{3}$ \\
\hline $\mathrm{MnO}_{2}$ & & & & & 159 & 0.58 & $4.0 \times 10^{2}$ & $3.5 \times 10^{3}$ \\
\hline LiMnO & & & & & 212 & 0.29 & $3.2 \times 10^{2}$ & $1.9 \times 10^{3}$ \\
\hline $\mathrm{Mn}_{2}$ & & & & & 263 & 0.27 & $7.1 \times 10^{2}$ & $2.4 \times 10^{3}$ \\
\hline
\end{tabular}

对于脉冲激光烧蚀 $\mathrm{LiMn}_{2} \mathrm{O}_{4}$ 产生的其他产物也进行了拟合. 表 1 列出一些主要烧蚀产物 经上述方法拟合分析得出的结果, 包括离子和中性产物飞行时间谱峰峰位时间 $\left(t_{m}\right)$, 质心速度 $(u)$, 最可几平动能 $\left(K_{E}\right)$ 和离开靶表面时的温度 $\left(T_{S}\right)$. 其中 $u$ 值和 $T_{S}$ 值由拟合 $(1)$ 或(2)式中的参数 $b$ 计算而得, $K_{E}$ 由 $0.5 m\left(L / t_{m}\right)^{2}$ 计算得出. 从表 1 可以看出: 虽然激光烧蚀 $\mathrm{LiMn}_{2} \mathrm{O}_{4}$ 靶产物的质 量相差很大, 但产物的平动能在同一个数量级, 这表明在膨胀前产物粒子已达到热平衡或局 部热平衡状态; 从拟合结果估计的粒子温度对原生离子为 $(1 \sim 6) \times 10^{4} \mathrm{~K}$, 中性产物为 $10^{3} \sim 10^{4} \mathrm{~K}$, 表明中性产物可能主要由靶表面物质的热蒸发过程产生, 而离子可能还有另外的生成和加速 机制 ${ }^{[13]}$; 离子产物的质心速度比中性产物的大一个数量级, 表明中性产物可能来源于激光脉 冲后热表面的蒸发, 或是源于等离子体从靶表面的部分反射, 这部分粒子处于膨胀等离子体 的后部分, 没有获得大的质心速度.

\section{2 激光能量密度的影响}

由于激光能量密度对烧蚀产物的质量分布和平动能的影响较大, 进一步测定了不同激光 能量密度下部分产物的 TOF 谱. 图 3 给出一些主要烧蚀产物 $\left(\mathrm{Li}, \mathrm{LiO}, \mathrm{LiO}_{2}\right.$ 及 $\left.\mathrm{Mn}\right)$ 的平动能与 激光能量密度间的关系. 可见, 在 $0.5 \sim 2.2 \mathrm{~J} \cdot \mathrm{cm}^{-2}$ 范围内上述烧蚀产物中原生离子的平动能基 本不随激光能量密度的增大而变化, 而中性产物的平动能随激光能量密度的增大略有增加. Hess ${ }^{[14]}$ 曾经指出, 激光能量密度与烧蚀产物平动能的关系可用于判别激光烧蚀是属于激光加 热过程还是非热的光化学过程. 对于光热过程, 由于加热表面温度随着激光能量密度的增大 而升高, 所以从表面烧蚀产生粒子的平动能将会随之增加; 而对于光化学解离和电离过程, 产 物的平动能仅取决于激光波长, 而与能量密度无关. 因此, 从上述结果可以认为 $\mathrm{LiMn}_{2} \mathrm{O}_{4}$ 激光 
烧蚀产生的中性产物可能是由光热过程产生的, 而离子主要通过非热的光解离和电离过程, 羽状物中烧蚀产物经绝热膨胀后已达到热平衡. 这些烧蚀产物彼此间动能大小的不同可能是 由于它们具有不同的质量引起的.
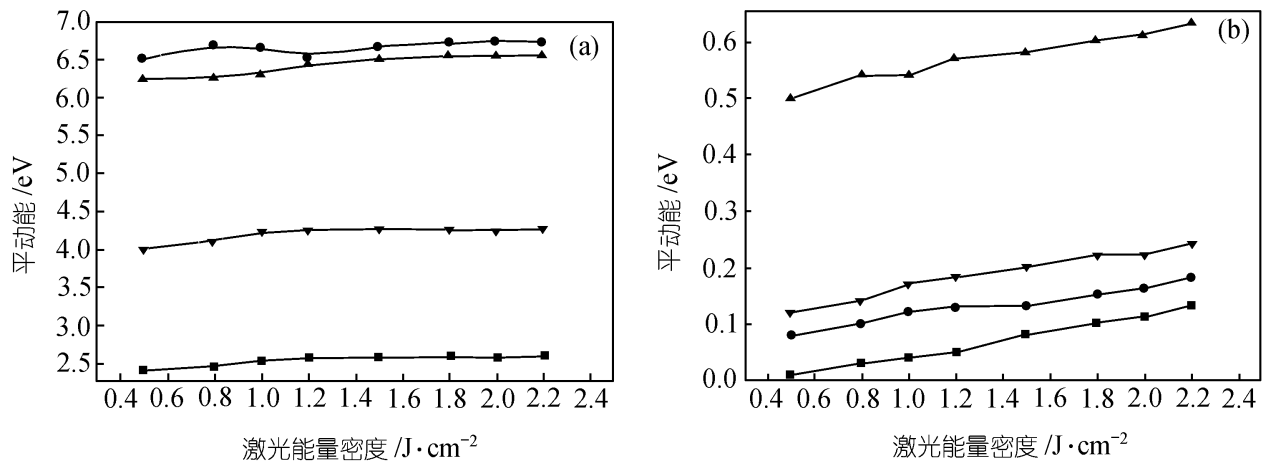

图 $3355 \mathrm{~nm}$ 脉冲激光烧蚀 $\mathrm{LiMn}_{2} \mathrm{O}_{4}$ 生成的离子产物(a) 和中性产物

(b)的最可几平动能与激光能量密度之间的关系

口示 $\operatorname{Li}(m / e=7)$,

- 示 $\mathrm{LiO}(m / e=23)$,

$\Delta$ 示 $\operatorname{Mn}(m / e=55)$,

示 $\mathrm{LiO}_{2}(m / e=39)$

表 $2355 \mathrm{~nm}$ 脉冲激光烧蚀 $\mathrm{LiMn}_{2} \mathrm{O}_{4}$ 靶, 产物中 $\mathrm{Li}, \mathrm{LiO}, \mathrm{LiO}_{2}$ 以及 $\mathrm{Mn}$ 的激光能量密度表观國值

\begin{tabular}{cccc}
\hline \multirow{2}{*}{ 产物 } & \multirow{2}{*}{$m / e$} & \multicolumn{2}{c}{ 激光能量密度表观國值 $/ \mathrm{J} \cdot \mathrm{cm}^{-2}$} \\
\cline { 3 - 4 } & & 原生离子 & 中性产物 \\
\hline $\mathrm{Li}$ & 55 & 0.37 & 0.25 \\
$\mathrm{Mn}$ & 23 & 0.46 & 0.36 \\
$\mathrm{LiO}$ & 39 & 0.55 & 0.41 \\
$\mathrm{LiO}_{2}$ & & 0.69 & 0.44 \\
\hline
\end{tabular}

在研究激光烧蚀氧化物时发现, 激光能量密度不但影响烧蚀产物的平动能以及信号强度, 而且对于不同的烧蚀产物往往存在不同的表观阈值. 我们通过测定激光烧蚀 $\mathrm{LiMn}_{2} \mathrm{O}_{4}$ 时不同 烧蚀产物的信号强度与激光能量密度的关系, 分别测得它们的激光能量密度表观國值, 见表 2 . 可以看出, 产生中性 $\mathrm{Li}$ 原子的表观阈值最低, 依次分别是 $\mathrm{Mn}, \mathrm{LiO}$ 和 $\mathrm{LiO}_{2}$; 离子产物 $\mathrm{Li}^{+}, \mathrm{Mn}^{+}$, $\mathrm{LiO}^{+}$和 $\mathrm{LiO}_{2}{ }^{+}$的表观阈值的大小规律与上述中性产物相似; 而各个离子产物的能量密度表观國 值都比相应的中性产物高.

\section{3 烧蚀产物的空间角分布}

考察脉冲激光烧蚀产物的空间角分布不仅有助于了解在基片上沉积薄膜的组成和厚度的 分布, 同时还能对激光烧蚀的机理提供有益的信息. 在激光能量密度为 $2.0 \mathrm{~J} \cdot \mathrm{cm}^{-2}$ 时, 测定了 原生锰离子和锰原子的空间角分布(见图 4). 由此图可见, 无论是金属 $\mathrm{Mn}$ 原子还是原生的 $\mathrm{Mn}^{+}$ 离子的空间角分布都强烈地集中在表面法线方向. Venkatesan ${ }^{[15]}$ 在研究 $\mathrm{Y}-\mathrm{Ba}-\mathrm{Cu}-\mathrm{O}$ 高 $\mathrm{T}_{\mathrm{C}}$ 薄膜时 认为, 生成激光烧蚀产物可以有两种通道: 一是热蒸发, 即通过激光加热在靶表面形成一高温 熔融区, 烧蚀产物飞向真空是通过热蒸发, 服从 $\cos \theta$ 分布(Knudsen 定律); 另一是非热过程, 即在激光对靶表面烧蚀产生的高密度气团内烧蚀产物经非热过程引发的向表面法线方向的

“次级喷射” (secondary ejection), 一般服从 $\cos ^{n} \theta$ 分布, 其中 $n$ 称为尖度参数(sharpness parameter). 但是在激光烧蚀过程中, 有些烧蚀产物的生成可以共存着上述两种通道, 其角分 布可用双余弦公式 $a \cos \theta+(1-a) \cos ^{n} \theta$ 表达. 图 4 中所列的烧蚀产物 $\mathrm{Mn}^{+}$和 $\mathrm{Mn}$ 的角分布经 
拟合所得的结果如下:

$$
\text { 原生 } \mathrm{Mn}^{+}: \cos ^{16} \theta \quad \mathrm{Mn} \text { 原子: } 0.19 \cos \theta+0.81 \cos ^{69} \theta,
$$

上述拟合结果表明: 对于原生的 $\mathrm{Mn}^{+}$主要通过非热过程产生, 而 $\mathrm{Mn}$ 原子的产生是热过程和非 热过程共同作用的结果, 其中热过程所占的比例 $f$ 可通过半球空间积分获得, 由下式 ${ }^{[19]}$ :

$$
f=\frac{(n+1) a}{a n-a+2},
$$

计算结果 $f=0.89$, 由此表明热过程占的比例为 $89 \%$, 非热过程为 $11 \%$. 这说明 $355 \mathrm{~nm}$ 脉冲激 光烧蚀 $\mathrm{LiMn}_{2} \mathrm{O}_{4}$ 产生的 $\mathrm{Mn}$ 原子主要通过热蒸发产生.
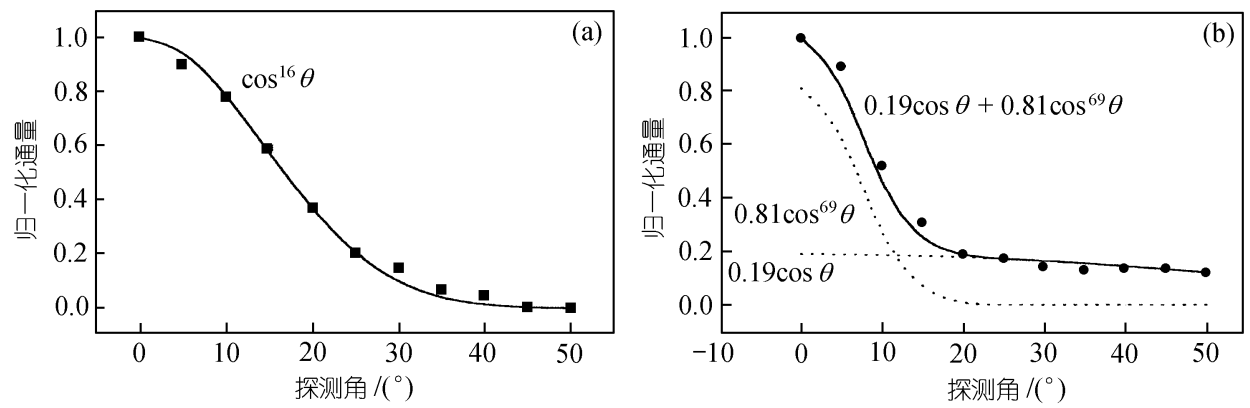

图 4 烧蚀产物 $\mathrm{Mn}$ 的空间角分布以及拟合结果

(a)电离灯丝关闭; (b)电离灯丝开启. 点为实验测量结果, 实线为拟合结果, 虚线分别为 $\cos \theta$ 以及 $\cos ^{n} \theta$ 的贡献

\begin{tabular}{|c|c|c|c|c|}
\hline \multirow{2}{*}{ 产物 } & \multicolumn{2}{|c|}{ 电离灯丝关闭 } & \multicolumn{2}{|c|}{ 电离灯丝开启 } \\
\hline & $0.8 \mathrm{~J} \cdot \mathrm{cm}^{-2}$ & $2.0 \mathrm{~J} \cdot \mathrm{cm}^{-2}$ & $0.8 \mathrm{~J} \cdot \mathrm{cm}^{-2}$ & $2.0 \mathrm{~J} \cdot \mathrm{cm}^{-2}$ \\
\hline $\mathrm{Li}$ & $\cos ^{18} \theta$ & $\cos ^{42} \theta$ & $\cos ^{12} \theta$ & $\cos ^{29} \theta$ \\
\hline $\mathrm{O}$ & $\cos ^{24} \theta$ & $\cos ^{35} \theta$ & $\cos ^{13} \theta$ & $\cos ^{51} \theta$ \\
\hline $\mathrm{LiO}$ & $\operatorname{Cos}^{9} \theta$ & $\cos ^{15} \theta$ & $\cos ^{10} \theta$ & $\cos ^{29} \theta$ \\
\hline $\mathrm{LiO}_{2}$ & $\cos ^{26} \theta$ & $\cos ^{68} \theta$ & $0.19 \cos \theta+0.81 \cos ^{50} \theta$ & $0.20 \cos \theta+0.80 \cos ^{72} \theta$ \\
\hline $\mathrm{Mn}$ & $\cos ^{15} \theta$ & $\cos ^{16} \theta$ & $0.23 \cos \theta+0.77 \cos ^{47} \theta$ & $0.19 \cos \theta+0.81 \cos ^{69} \theta$ \\
\hline $\mathrm{Li}_{2}$ & & & $0.18 \cos \theta+0.82 \cos ^{58} \theta$ & $0.21 \cos \theta+0.79 \cos ^{85} \theta$ \\
\hline $\mathrm{Li}_{4}$ & & & $0.22 \cos \theta+0.78 \cos ^{70} \theta$ & $0.27 \cos \theta+0.73 \cos ^{105} \theta$ \\
\hline $\mathrm{Li}_{6}$ & & & $0.31 \cos \theta+0.69 \cos ^{70} \theta$ & $0.35 \cos \theta+0.65 \cos ^{58} \theta$ \\
\hline LiMn & & & $0.26 \cos \theta+0.74 \cos ^{25} \theta$ & $0.20 \cos \theta+0.80 \cos ^{39} \theta$ \\
\hline $\mathrm{MnO}$ & & & $0.32 \cos \theta+0.68 \cos ^{28} \theta$ & $0.27 \cos \theta+0.73 \cos ^{41} \theta$ \\
\hline $\mathrm{MnO}_{2}$ & & & $0.39 \cos \theta+0.61 \cos ^{34} \theta$ & $0.27 \cos \theta+0.73 \cos ^{54} \theta$ \\
\hline
\end{tabular}

表 3 不同的激光能量密度下 $355 \mathrm{~nm}$ 脉冲激光烧蚀 $\mathrm{LiMn}_{2} \mathrm{O}_{4}$ 产物的空间角分布的拟合结果

$355 \mathrm{~nm}$ 脉冲激光烧蚀 $\mathrm{LiMn}_{2} \mathrm{O}_{4}$ 靶的其他主要产物与 $\mathrm{Mn}(m / e=55)$ 具有类似的角分布, 它 们也可以通过 $\cos ^{n} \theta$ 以及 $a \cos \theta+(1-a) \cos ^{n} \theta$ 拟合. 表 3 列出了在激光能量密度为 0.8 和 2.0 $\mathrm{J} \cdot \mathrm{cm}^{-2}$ 时一些烧蚀产物的角分布拟合结果. 可见, 当电离灯丝关闭时, 所有的烧蚀产物均可用 $\cos ^{n} \theta$ 拟合, 而当电离灯丝开启时, 许多产物采用双余弦函数拟合比较满意; 从整体上说, 随着 激光能量密度的增加, 非热过程 $\cos ^{n} \theta$ 中的尖度参数 $n$ 值增大, 但热过程所占的比例的变化却 比较复杂. 激光能量密度对烧蚀产物角分布的影响, 可能由于在高能量密度下激光烧蚀产物 的密度较高, 增加了粒子间的平均碰撞数, 最终使出射束流在靶表面的法线方向越加集中, 尖 度参数 $n$ 也越大. 


\section{3 结论}

利用角分辨飞行时间质谱法研究了 $355 \mathrm{~nm}$ 脉冲激光烧蚀 $\mathrm{LiMn}_{2} \mathrm{O}_{4}$ 的反应, 激光能量密度 较大时, 烧蚀产物中的氧化物不仅相对量增加, 而且物种更加丰富. 由此表明较高能量密度的 激光烧蚀 $\mathrm{LiMn}_{2} \mathrm{O}_{4}$ 对于团簇和其他化合物分子的形成是有利的. 虽然 $355 \mathrm{~nm}$ 脉冲激光烧蚀 $\mathrm{LiMn}_{2} \mathrm{O}_{4}$ 靶产物的质量相差很大, 它们的飞行时间谱可用带质心速度的 Maxwell-Boltzmann 分 布函数拟合. 离子产物的平均动能与激光能量密度无关, 而中性产物的平动能随激光能量密 度的增大略有增加. 说明 $\mathrm{LiMn}_{2} \mathrm{O}_{4}$ 激光烧蚀产生的中性产物可能是由光热过程产生的, 而离 子主要通过非热的光解离和电离过程, 羽状物中烧蚀产物经绝热膨胀后已达到热平衡. 另外, 激光烧蚀的产物 $\mathrm{Li}, \mathrm{LiO}, \mathrm{LiO}_{2}$ 和 $\mathrm{Mn}$ 存在表观能量密度國值, 离子产物的表观能量阈值比相 应的中性产物高. 烧蚀产物中原生离子和中性产物的空间角分布可以用 $\cos ^{n} \theta$ 或 $a \cos \theta+(1-a)$ $\cdot \cos ^{n} \theta$ 拟合. 随着激光能量密度的增加, 非热过程 $\cos ^{n} \theta$ 中的尖度参数 $n$ 值增大, 但热过程所 占的比例的变化却比较复杂.

\section{参考文献}

1 Balkanski M. Solid-state microbatteries for electronics in the 21st century. Solar Energy Materials \& Solar Cells, 2000, 62: $21 \sim 35$

2 Winter M, Besenhard J O, Spahr M E, et al. Insertion electrode materials for rechargeable lithium batteries. Adv Mater, 1998, 10(10): 725 750

3 Julien C, Haro-Poniatowski E, Camacho-Lopez M A, et al. Growth of $\mathrm{LiMn}_{2} \mathrm{O}_{4}$ thin films by pulsed laser deposition and their electrochemical properties in lithium microbatteries. Materials Science and Engineering B, 2000, 72: 36 46

4 Striebet K A, Deng C Z, Wen S J, et al. Electrochemical behavior of $\mathrm{LiMn}_{2} \mathrm{O}_{4}$ thin films produced with pulsed laser deposition. J Electrochem Soc, 1996, 143: 1821 1827

5 Rougier A, Striebel K A, Wen S J, et al. Cyclic voltammetry of pulsed laser deposited $\mathrm{LiMn}_{2} \mathrm{O}_{4}$ thin films. J Electrochem Soc, 1998, 145: 2975 2980

6 Fu Z, Kong J, Qin Q. Electrochemical and electrochromic properties of niobium oxide thin films fabricated by pulsed laser deposition. J Electrochem Soc, 1999, 146: 3914 3918

7 Zhou M, Fu Z, Zhang L, et al. Structure and stability of LiOMn molecule generated by laser ablation. Chem Phys Lett, 2000, 318: $644 \sim 648$

8 党海军, 韩镇辉, 秦启宗. 激光烧蚀 La-Ca-Mn-O 反应产物的角分辨和时间分辨质谱研究. 中国科学, A 辑, 1998, 28(10): 930 935

9 Dang H, Han Z, Dai Z, et al. Characterization of laser ablated species from a La-Ca-Mn-O target by angle- and time-resolved mass spectrometry. Int J Mass Spectrom Ion Process, 1998, 178: 205 212

10 Tang Y, Qin Q. Mass, velocity and angular distributions of the species ejected from laser ablation of $\mathrm{LiCoO}_{2}$. Chem Phys Lett, 2001, 343: 452 457

11 Qin Q, Zhou M, Mao D. Time-of-flight mass spectrometric study on UV laser ablation of silver chloride. Appl Surf Sci, 1997, 119: $321 \sim 329$

12 卢平和, 秦启宗. 飞行时间法研究激光诱导金属表面蚀刻反应动力学. 中国科学, A 辑, 1993, 23(6): 650 656

13 Dang H, Qin Q. A thermal-controlling mechanism for laser ablation of a colossal magnetoresistant oxide target. Chem Phys Lett, 2002, 354: 210 216

14 Hess P. Time-of-flight analysis of IR and UV laser-induced multilayser desorption and ablation. In: Topic in Current Physics. Berlin: Springer-Verlag, 1998. 55 60

15 Venkatcsan T, Wu X D, Inam A, et al. Observation of two distinct components during pulsed laser deposition of high Tc super conducting films. Appl Phys Lett, 1988, 52 (14): 1193 1195 